\title{
Morphological Features of the Most Advanced Intra-Tumor Component in Multistep Progression
}

\begin{abstract}
Advances in basic life science during the past decade have exponentially increased our understanding of the molecular bases of lung cancers and this improved insight has had great effects on diagnosis, prognosis, classification, and treatment of cancer. Similar to colorectal carcinoma, stepwise development of lung cancer has been proposed. For squamous neoplasm, dysplasia is considered to progress to invasive cancer through carcinoma in-situ in association with accumulations of genetic and epigenetic alterations. In lung adenocarcinoma, a preinvasive lesion, termed atypical adenomatous hyperplasia, progresses to an adenocarcinoma in situ, namely a bronchioloalveolar carcinoma, followed by an invasive adenocarcinoma. Currently, molecular alterations, which are involved in each transition, have been also revealed. In this review, I would like to discuss correlation of morphological features with the progression of lung adenocarcinoma with special reference to the most advanced component within the tumor. (J Lung Cancer 2010;9(2):53 $-56)$
\end{abstract}

Key Words: Lung neoplasms, Disease progression, Cell morphology, Epidermal growth factor receptor
Yasushi Yatabe, M.D.

Department of Pathology and Molecular Diagnosis, Aichi Cancer Center, Nagoya, Japan

Received: November 15, 2010 Accepted: November 20, 2010

\section{Address for correspondence}

Yasushi Yatabe, M.D. Department of Pathology and Molecular Diagnosis, Aichi Cancer Center, Nagoya 464-8681, Japan

Tel: 81-52-762-6111

Fax: 81-52-757-4810

E-mail: yyatabe@aichi-cc.jp
Generally, tumor grade or differentiation is determined by the combination of structural and cellular atypia. The structural and cellular characteristics of a tumor are correlated in most instances, but they can diverge. Although pathologists share a vague consensus concerning which atypia should be considered more significant according to the tumor type, there are two basic notions. One is that the grade of the tumor should be determined by the most prevalent grade, while the other is that the tumor grade should be determined by the highest grade in the tumor. In the case of lung adenocarcinoma mixed with components of small cell lung cancer (SCLC), the tumor grade is determined as grade 4 regardless of the extent of the SCLC component (1). Indeed, combined SCLC/adenocarcinoma tumors frequently have metastases consisting solely of SCLC components. Because the component that is most advanced along the progression schema has the greatest chance of metastasis and/or clinical aggressiveness, we must decide how we should identify the most advanced component within a tumor morphologically.
Fig. 1A and B show two components in a single nodule of lung adenocarcinoma; Fig. 1A shows a tissue with a solid growth pattern partly with cribriform growth, while the acinar component is predominant in the tissue shown in Fig. 1B. Structurally, a solid growth pattern is considered to be a higher grade than an acinar pattern. In contrast, the cellular atypia in this tumor is greater in the acinar component than in the solid component based on nuclear irregularity and the chromatin pattern. Which component should be considered the most clinically important and deserving of a higher grade?

In general, it is accepted that a poorly differentiated portion of the tumor or the portion with the highest grade is the component that is the most advanced. In endometrial cancer, the histological grade is determined by the extent of the solid growth pattern, and a grade 3 tumor is defined as an endometrial carcinoma with $50 \%$ or more solid growth (2). However, when notable nuclear atypia is seen, the tumor is evaluated as grade 3 regardless of the proportion of solid growth. Thus, endometrial carcinoma is graded primarily based 


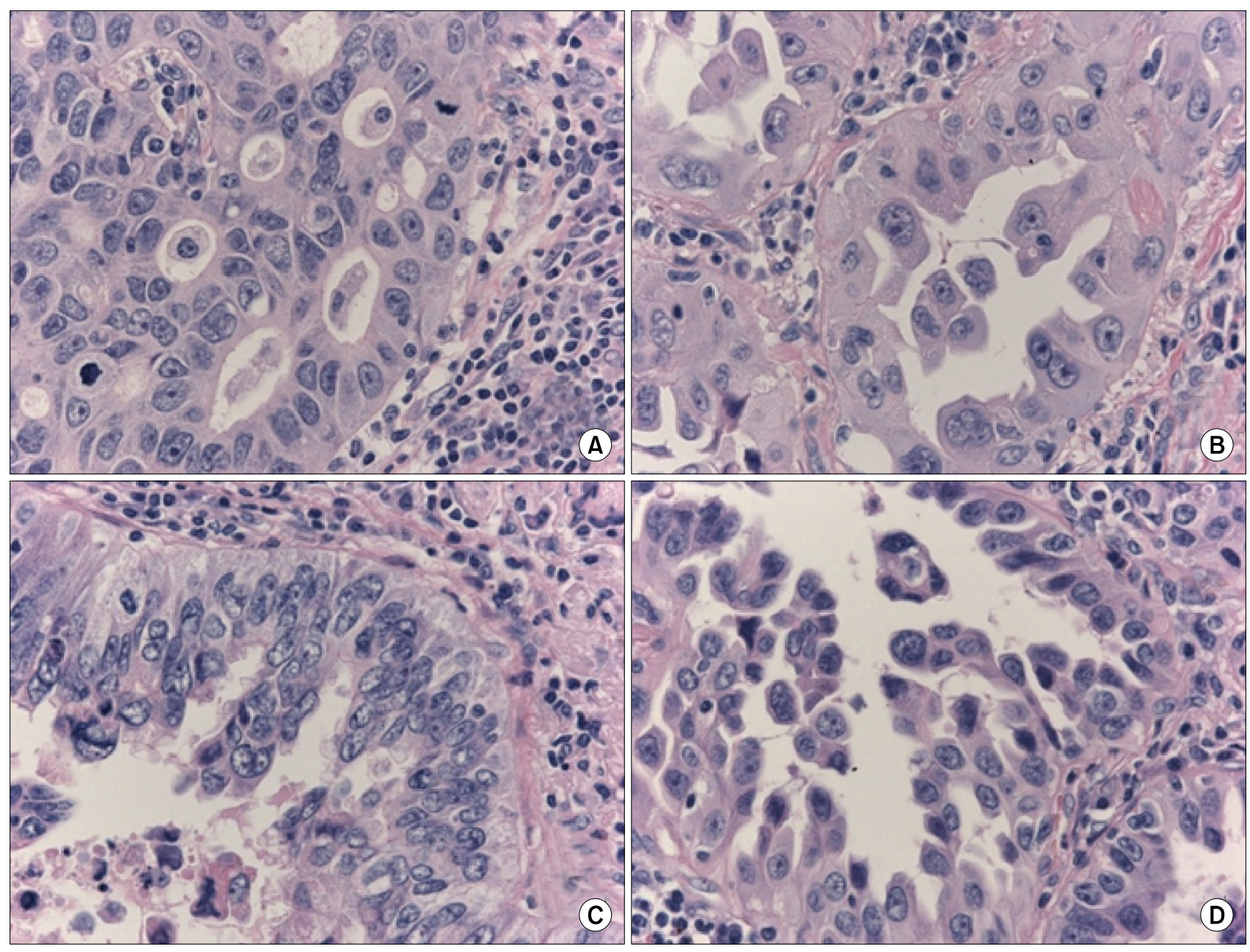

Fig. 1. Four tissue samples from a single tumor. Morphologically, the four components were different with respect to the growth pattern and cellular atypia. All of the four components (A to D) harbored an epidermal growth factor receptor (EGFR) mutation, but EGFR amplification was detected only in components (B) and (D).

on structural atypia, although cellular atypia are partly taken into consideration. In breast cancer, the Nottingham histological grading system is widely used $(3,4)$, and this system is based on three features of the tumor cells: tubular formation, nuclear atypia and mitotic count. This system regards the structural and cellular atypia as equally significant. In lung cancer, the 2004 WHO classification does not define tumor differentiation or grade because of the lack of reproducibility and clinical significance (1). However, the solid growth component is of a higher grade than the papillary component, according to the general consensus of tumor grading. Therefore, the tumor portion with a solid growth pattern (Fig. 1A) appears to be more advanced along the cancer progression schema than the component with papillary growth (Fig. 1B).

On the other hand, there is a paradigm in modern molecular oncology that cancer progresses in a multi-step fashion. This concept was introduced by Vogelstein et al., and the progression of hyperplasia from adenoma to carcinoma is promoted by the accumulation of genetic alterations (5). According to this concept, a part of the adenoma acquires the genetic alterations that drive the transformation to invasive carcinoma. Therefore, the portion of the tumor with a greater number of accumulated genetic alterations represents the part that is most advanced. Using this distinction, we can clarify some morphological differences that are associated with cancer progression. Indeed, such a correlation between tumor progression and morphological characteristics has been reported. A soft tissue tumor, dermatofibrosarcoma protuberans, which harbors the characteristic COL1A1-PDGFB fusion gene, occasionally transforms into high-grade fibrosarcoma. Recently, 

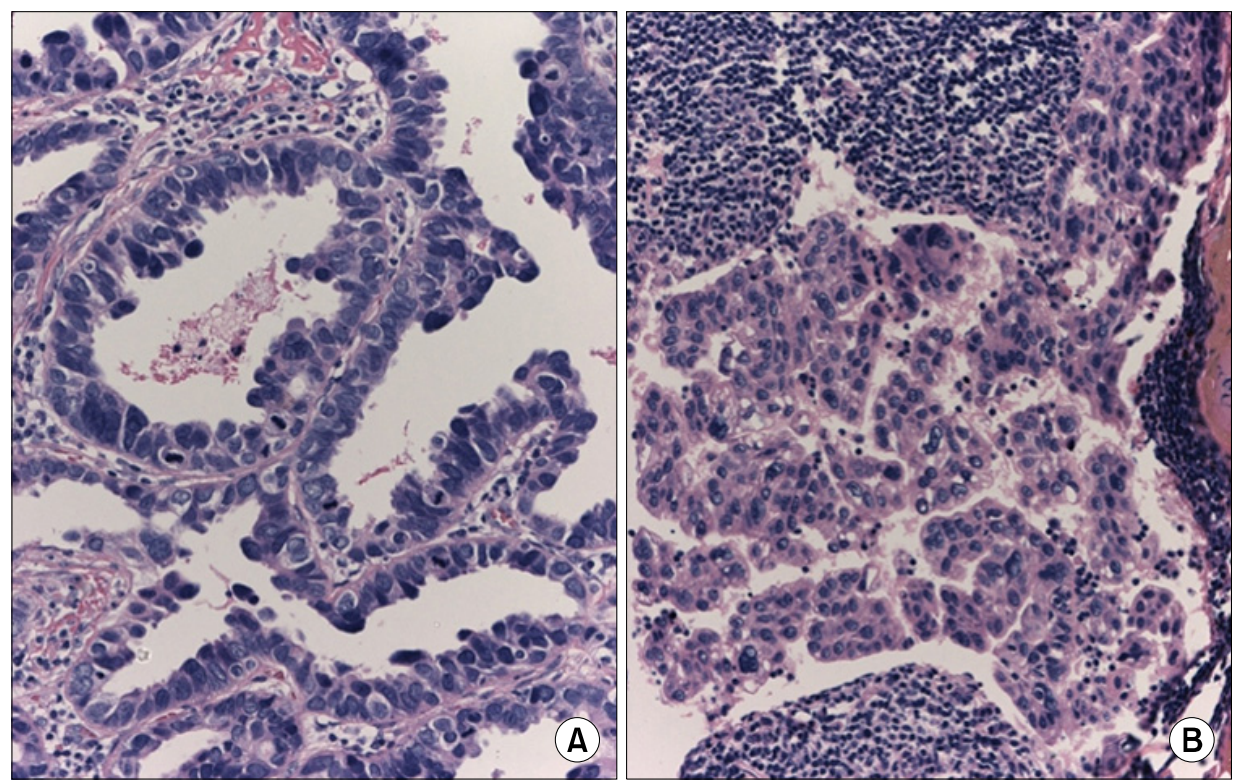

Fig. 2. The primary tumor (A) and the metastasis in the lymph node (B) in a patient treated with gefitinib. A secondary mutation, T790M, was superimposed only in the regrown primary tumor $(A)$; the L858R point mutation was detected in both tumors.

it has been shown that a copy number gain of the fusion gene is present in the high-grade lesion, in addition to the translocation (6).

We recently proposed a hypothesis concerning genetic alterations of epidermal growth factor receptor (EGFR) (7). Within a tumor, EGFR is mutated uniformly, while EGFR amplification is heterogeneously distributed. Detailed examination revealed that EGFR amplification was detected exclusively in invasive and/or high-grade areas. In addition, when the frequencies of the EGFR amplification according to the progression steps from precancerous lesion to invasive cancer were compared, EGFR was disproportionally amplified among the progression steps, although EGFR mutation rates showed almost equal frequencies. These findings suggested that the EGFR mutation is involved in the very early steps of progression, whereas EGFR amplification is acquired in association with tumor progression. These findings have been confirmed by others $(8,9)$. Therefore, a proportion of a tumor exhibiting EGFR amplification could be regarded as indicative of a lesion that is more advanced.

Accordingly, EGFR mutations and amplification were examined in each of the tumor portions shown in Fig. 1A and B. The cribriform/solid component (Fig. 1A) harbored an EGFR mutation but not EGFR amplification. In contrast, both EGFR mutation and amplification were detected in the acinar component, which had a higher level of nuclear atypia (Fig. 1B). This result suggests that the acinar component is more advanced than the cribriform/solid component. We further examined various morphological components and found that morphological changes generally corresponded to EGFR amplification; however, there were differences in the EGFR amplification even among morphologically identical components. Fig. 1C and D are examples of such tissues.

Similarly, Fig. 2 shows a lung adenocarcinoma that harbors a resistance mutation after EGFR-tyrosine kinase inhibitor treatment. This patient, who had cT2NOM0 lung adenocarcinoma, was treated with gefitinib because treatment with cytotoxic drugs was refused. This treatment was very effective, especially in the mediastinal lymph nodes, but two months later, the primary tumor began to regrow. Surgical resection and lymph node dissection were completed. The hot spot point mutation L858R was detected in both the primary nodule and in the metastatic tumor in the lymph node, but a resistance mutation, T790M, was superimposed only in the regrown primary tumor. According to the progression schema, the primary tumor with the secondary T790M mutation is likely to be the portion that is more advanced (10). Are the morphological features in Fig. 2 consistent with this genetic difference?

The results appear to suggest that morphological features are not always associated with genetic differences. However, I do not consider this conclusion to be the result of the inferior capability of pathologists; it is simply the result of using inadequate morphological indicators. If adequate indicators 
were obtained, our pathologists could integrate morphologybased wisdom with molecular biology-based knowledge that directly connects to clinical treatment.

\section{REFERENCES}

1. Travis WD. Pathology and genetics of tumours of the lung, pleura, thymus, and heart. 15th ed. Lyon: IARC Press; 2004.

2. Zaino RJ, Kurman RJ, Diana KL, et al. The utility of the revised International Federation of Gynecology and Obstetrics histologic grading of endometrial adenocarcinoma using a defined nuclear grading system. A Gynecologic Oncology Group study. Cancer 1995;75:81-86.

3. Le Doussal V, Tubiana-Hulin M, Friedman S, et al. Prognostic value of histologic grade nuclear components of ScarffBloom-Richardson (SBR). An improved score modification based on a multivariate analysis of 1262 invasive ductal breast carcinomas. Cancer 1989;64:1914-1921.

4. Elston CW, Ellis IO. Pathological prognostic factors in breast cancer. I. The value of histological grade in breast cancer: experience from a large study with long-term follow-up.
Histopathology 1991;19:403-410.

5. Fearon ER, Vogelstein B. A genetic model for colorectal tumorigenesis. Cell 1990;61:759-767.

6. Abbott JJ, Erickson-Johnson M, Wang X, et al. Gains of COL1A1-PDGFB genomic copies occur in fibrosarcomatous transformation of dermatofibrosarcoma protuberans. Mod Pathol 2006;19:1512-1518.

7. Yatabe Y, Takahashi T, Mitsudomi T. Epidermal growth factor receptor gene amplification is acquired in association with tumor progression of EGFR-mutated lung cancer. Cancer Res 2008;68:2106-2111.

8. Sholl LM, Yeap BY, Iafrate AJ, et al. Lung adenocarcinoma with EGFR amplification has distinct clinicopathologic and molecular features in never-smokers. Cancer Res 2009;69: 8341-8438.

9. Tang X, Varella-Garcia M, Xavier AC, et al. Epidermal growth factor receptor abnormalities in the pathogenesis and progression of lung adenocarcinomas. Cancer Prev Res (Phila) 2008;1:192-200.

10. Suda K, Onozato R, Yatabe Y, et al. EGFR T790M mutation: a double role in lung cancer cell survival? J Thorac Oncol 2009;4:1-4. 